\title{
La relación entre las opiniones acerca de la docencia universitaria y la función tutorial del profesor en la universidad
}

\author{
Ethel Trengove Thiele*
}

\begin{abstract}
RESUMEN
Esta investigación pretende indagar en la opinión que tienen las estudiantes de $4^{\circ}$ año de la carrera de Educación Diferencial, en cuanto al desempeño profesional de la investigadora en diferentes ámbitos de la docencia, tales como la organización y estructura de la asignatura, es decir, si se evidencia suficiente dominio teórico y conceptual del tema que se desarrolla en la programación; si presenta una actitud mediadora para generar los propios aprendizajes de los estudiantes. Complementario a lo anterior, indagar si las estrategias de enseñanza son pertinentes a los aprendizajes esperados. Por último, saber si los procedimientos evaluativos son pertinentes y se enfocan a los aprendizajes que han alcanzado los estudiantes, y cuál es la percepción en torno a la interacción y clima que se genera dentro de las propias clases, y más allá del aula. Todo esto, con la finalidad de relacionarlo con prácticas pedagógicas innovadoras, como lo es la función tutorial del docente universitario, detectando aquellos puntos valorados y los no tan valorados para proyectar mejoras en aquellos aspectos que así lo requieran.
\end{abstract}

Palabras clave: Competencias docentes, organización curricular, estrategias de enseñanza, procedimientos de evaluación.

\section{Relationship between opinions about University teaching and the mentoring role of the University teacher}

\begin{abstract}
This research aims to researching opinions that Fourth-year students of Special Education have regarding to the professional performance of the researcher in different teaching fields such as organization and structure of a course, that is to say, whether there is evident sufficient theoretical and conceptual mastery of the subject, whether there is a mediating attitude to generate learning in students. Complementary to that, the paper is aimed to reviewing whether teaching strategies are relevant to the learning objectives. Finally, it seeks to know whether the evaluation procedures are relevant and focused on learning students have achieved, and what would be the perception around interactions and climates generated in the classroom and beyond. The objective is to create a relationship with innovative teaching practices, as the mentoring role of the University teacher, unveiling valued and not valued aspects in order to project improvements in those weak areas.
\end{abstract}

Keywords: Teaching skills, curricular organization, teaching strategies, evaluation procedures and interactions between teacher and student.

Profesora de Educación Diferencial, Magíster en Gestión Educacional. Académica de la Escuela de Educación Diferencial de la Universidad Católica Silva Henríquez. Chile. E-mail: etrengovet@ucsh.cl 


\section{Introducción}

Esta propuesta de Trabajo de Fin de Máster (TFM) nace desde el interés de revisar mi propia docencia en una asignatura que se dicta en el último año que cursan los estudiantes de Pedagogía en Educación Diferencial en la Universidad Católica Silva Henríquez (en adelante UCSH), con el objetivo de conocer sus opiniones, detectar los aspectos más y menos valorados de la docencia universitaria, y relacionarlo con la función tutorial del profesor en la universidad.

Como señala Zabalza (2009), ser docente implica un compromiso con lo educativo, y también con la técnica didáctica, es decir, no solo el cumplimiento de lo que se propone en el programa de la asignatura, si no que la forma en que se presenta ese conocimiento, de manera tal que sea accesible para todos, sin segregar por condiciones culturales, sociales o personales. La meta del TFM será conocer estas opiniones, analizarlas a la luz de los objetivos planteados y de dimensiones previamente estipuladas a partir de la literatura asociada a la función tutorial universitaria como estrategia de innovación docente, dando pie a la propia reflexión crítica para implementar mejoras en las futuras asignaturas; "Hemos de recordar que lo educativo constituye hoy día uno de los espacios de reflexión, análisis, confrontación y toma de posturas más vivas y concurridas de nuestro panorama social” (Zabalza, 2009, p. 9).

La revisión bibliográfica y cuerpo teórico estará centrado en la literatura que permita sustentar la investigación y dar respuestas a las preguntas y objetivos planteados. Fundamentalmente, en temáticas en relación con competencias docentes, organización curricular, estrategias de enseñanza; tutorías, evaluación y las interacciones entre los actores.

El marco metodológico se enmarca en un enfoque cualitativo, pues la intención es analizar para comprender la realidad in situ de lo que se está desarrollando en el quehacer docente, en el que se utiliza una metodología mixta de recogida de datos, basada en el análisis documental y de la información recopilada a través de una entrevista y un cuestionario, lo que permitirá tabular los datos determinando una visión cuantitativa inicial, para luego profundizar en un análisis 
interpretativo realizado en matrices valorativas y de constructo, para finalizar con las conclusiones que permitirán proyectar las mejoras que requiero en mi práctica docente. Su diseño corresponde a un estudio de caso, con un universo constituido por el grupo curso completo de la asignatura Propuestas de Intervención en la Discapacidad, que involucra I5 estudiantes.

\section{Objetivos de la investigación}

\section{Objetivo general:}

Analizar las opiniones vertidas por estudiantes que cursan $4^{\circ}$ año en Educación Diferencial de la UCSH, acerca de la docencia universitaria de la asignatura Propuesta de Intervención en la DI y su relación con la función tutorial del profesor en la universidad.

\section{Objetivos específicos:}

- Identificar los elementos organizativos de la estructura curricular que perciben las estudiantes de la asignatura.

- Determinar el nivel de dominio teórico que identifican las estudiantes en la docente que desarrolla la asignatura.

- Describir las estrategias de enseñanza que identifican las estudiantes en la práctica pedagógica de la docente de la asignatura.

- Caracterizar los diferentes elementos evaluativos que identifican las estudiantes, como utilizados por la docente de la asignatura.

- Identificar los tipos de interacción que se establecen entre docente y alumnos más allá de la docencia de aula.

- Determinar aquellos aspectos susceptibles de mejorar a partir de la relación con la función tutorial del profesor en la universidad.

\section{Cuerpo teórico del estudio:}

\section{Competencias del docente universitario}

Zabalza define "las competencias docentes como esa batería de conocimientos, habilidades y actitudes que nos capacitarán para el 
buen desempeño de la profesión docente” (2009, p. 78), es decir, un conjunto de elementos que atañen a los aspectos disciplinares, pero también el cómo este profesional se enfrenta a la acción de provocar aprendizajes y se centra más en lo que los estudiantes deben aprender, por sobre el cómo él debe enseñar.

Por lo tanto, un profesor idealmente debiera transitar desde una docencia centrada en lo que debe hacer, a una docencia centrada en lo que debiera provocar en los estudiantes; tal como plantea Bain (2008) se "distingue entre una idea tradicional de enseñanza basada en contar a los estudiantes lo que el profesor sabía ("yo hablo, los estudiantes atienden y toman apuntes de lo que digo”, en palabras de un profesor) de una visión nueva de la enseñanza: "crear un entorno de aprendizaje estimulante en el que los estudiantes puedan tener control de su propio aprendizaje y que aprendan en el proceso con mayor profundidad". (Bain, 2009, pp. II-I6, citado en Zabalza, 2009, pp. 75-76)

Lo planteado en los párrafos anteriores se percibe en dos paradigmas relacionados con la docencia; uno que refleja la labor docente como la mera transmisión de información y otro que refleja la labor del docente como un guía, mediador y orientador; de manera muy clara, "independientemente de las teorías del aprendizaje que asumamos, nuestra tarea como formadores radica en el diseño de ambientes de aprendizaje que ayuden a nuestros alumnos a aprender" (Murillo. 2006, p. 50), es decir, aquellos docentes que se inclinan por este paradigma son quienes buscan desarrollar espacios de aprendizaje basados en el descubrimiento, experimentación e innovaciones docentes; "la formación de estudiantes no se limita únicamente a la adquisición de conocimientos y destrezas, sino que atiende también tanto al campo de las actitudes y disposiciones, como a la reflexión y acción colaborativa” (Mayor, 2010, p. 4).

\section{Organización curricular}

La carrera de Educación Diferencial es parte de las carreras que se dictan dentro de la Facultad de Educación de la Universidad Católica Silva Henríquez, cuyo origen se remonta al año 1982. Otorga actualmente el título de profesor de Educación Diferencial 
con Mención en Discapacidad Cognitiva y Alteraciones Severas del Desarrollo o en Dificultades del Aprendizaje e Inclusión Educativa, más el grado académico de Licenciado en Educación (Informe de Autoevaluación CNA, 20I4)

En su malla curricular tiene organizadas asignaturas que responden a las demandas del medio, una de las actividades mínimas y obligatoria se dicta en el nivel 700, equivalente al $4^{\circ}$ año y es "Propuesta de Intervención en la Discapacidad”, cuyo descriptor señala que dicha "actividad curricular tiene como propósito la determinación de los apoyos psicopedagógicos necesarios a partir de un estudio contextuado de las necesidades educativas asociadas a la discapacidad cognitiva" (Formulario NB-4. Didoc, 2009, p. I)

Esta asignatura mínima y nuclear dentro de la formación se organiza en 3 unidades; las primeras son fundamentalmente teóricas y se desarrollan en la universidad y la $3^{\text {a }}$ se complementa con trabajo en terreno, es decir, primero genera bases teóricas para luego aplicarlas in situ, ya que "para ser profesor universitario es condición sine qua non dominar el saber y el saber hacer de la disciplina, pero desde la óptica del cómo se genera dicho saber (investigación)" (Saravia, 20I I, p. 7).

La Society for Teaching and Learning in Higher Education (1996) plantea la existencia de dos principios éticos para el profesor universitario, uno de ellos es que debe tener un conocimiento exhaustivo de la disciplina que enseña, y el otro se relaciona con la competencia pedagógica; "El profesor pedagógicamente competente comunica los objetivos de su curso a sus estudiantes, es conocedor de la existencia de métodos y estrategias alternativas y selecciona aquel método de instrucción que, de acuerdo con las evidencias de investigación, resulta más efectivo para ayudar a sus estudiantes a alcanzar los objetivos del curso" (Zabalza, 2009, pp. 79-80); es decir y desde el punto de vista planteado, la organización y planificación de los aprendizajes pasa a tener una relevancia mucho más connotada de lo que habitualmente se considera, pues es parte del desempeño ético de todo docente tener claro en qué debe hacer, para qué lo va a hacer, cómo lo va a hacer y cómo lo va a evaluar, para confirmar si se logró o no la meta de aprendizaje propuesta. 
El docente debiera ser provocador, generador, diseñador de los ambientes de aprendizaje; no es transmisor de conocimiento, pues lo que se busca es que los estudiantes sean curiosos, motivados, autónomos y responsables de su propia formación. En el caso particular de este estudio, se podrán conocer las opiniones acerca de algunos de los aspectos señalados, pero para que tenga un real impacto en la docencia se relacionará con elementos de la planificación tutorial y de cómo esta estrategia de innovación facilitaría la tarea de aprendizaje en los estudiantes de la asignatura.

\section{Estrategias de enseñanza; función tutorial}

Como docentes, es imprescindible innovar en el quehacer pedagógico, incorporando diversas estrategias de trabajo que tengan como protagonistas a los estudiantes que se encuentran en proceso de formación, pues no hay manera más significativa que aprender haciendo. Para ello es importante planificar con antelación, y a la vez crear secuencias de actividades que se ajusten a la diversidad de estudiantes, en el sentido de sus requerimientos, necesidades y competencias, siendo diseñadas por el docente desde la mirada formativa y sin olvidar en ningún momento que "es el alumnado quien construye su conocimiento a partir de actividades de aprendizaje” (Marcelo, Yot \& Mayor, 20II, p. 38).

Dentro de la mencionada planificación de la clase, creación de la secuencia y determinación de las estrategias, el objetivo debiera ser enseñar a aprender, en donde el docente tome un rol de mediador, que sea quien guíe a los estudiantes y asuma un rol de facilitador en este proceso complejo; como señalan Marcelo et al., "los profesores universitarios deben concebir la enseñanza como fomento del aprendizaje y deben crear un entorno de aprendizaje crítico natural donde las personas aprendan enfrentándose a problemas importantes, atractivos e intrigantes"(20I I, p. 38).

Para efectos de este estudio, la estrategia que se profundizará por su significancia para el estudiante es la tutoría, que de acuerdo al Dr. Zabalza es "entendida como el conjunto de dispositivos que la institución universitaria debe establecer como sistema de orientación 
y apoyo a sus estudiantes" (Zabalza, 2013, p. II). Lo anterior implica que la universidad debe generar los espacios de aprendizaje para todos los estudiantes que en ella ingresen y que se sientan motivados, siendo el docente un eje central para esta acción, cumpliendo una función tutorial que va más allá de un mero acompañamiento; implica asumir un rol de guía, orientador y mediador, y estar con una disposición a favorecer el desarrollo de sus estudiantes, inclusive fuera del aula: "ser docentes es mucho más que ser enseñantes” (Zabalza, 20I3, p. I3).

Entre las formas cómo es entendida la tutoría se destaca la siguiente: "conjunto de actuaciones dirigidas a apoyar el pleno desarrollo personal y académico del estudiante universitario, se considera, cada vez más, como un indicador de calidad. Bien organizada, contribuye a mejorar la adaptación y rendimiento de los estudiantes a la vez que mejora la imagen pública de la universidad" (Gairín, Feixas, Franch, Guillamón \& Quinquer, 2004, p. 2I). Por tanto, la labor docente es acompañar y dar orientaciones para que el proceso formativo sea exitoso y operativo para la futura incorporación profesional al mundo laboral. Esta mirada activa del aprendizaje se complementa sin dificultades con las tradicionales clases magistrales que dictamos los docentes.

\section{Rol o funciones del tutor en la universidad}

De acuerdo a los autores revisados, "El profesor tutor es aquel que tiene especialmente encomendado a un estudiante (o grupo de estudiantes) para asesorarlo y ayudarlo en su proceso educativo, como medio de contribuir en el desarrollo de las competencias genéricas y disciplinares definidas para cada titulación. Es el profesor, que a la vez y en convergencia con las funciones de docencia, lleva a cabo un conjunto de actividades orientadoras y formativas, procurando que el estudiante alcance el máximo desarrollo en lo cognitivo, lo personal, lo académico y lo profesional" (García et al., 2005, p. I9I).

Lo anterior quiere decir que la responsabilidad docente ha aumentado así como ha aumentado la información y el acceso a la misma: ya no se trata de transmitir nada como conocimiento exclusivo del docente, sino que de generar en los estudiantes el interés, la motivación y la 
curiosidad por acceder a información de la web u otros espacios no necesariamente virtuales, que complementen sus saberes y le abran puertas a nuevos conocimientos, en la medida en que se van generando nuevas necesidades: el tutor debe transmitir al estudiante que el aprendizaje es una actividad dinámica y que se debe actualizar de manera permanente.

Es posible entonces definir "la tutoría universitaria como una actividad formativa realizada por el profesor-tutor encaminada al desarrollo integral -intelectual, profesional y humano- de los estudiantes universitarios" (García et al., 2005, p. I9I).

En cuanto a los roles, tanto del profesor como del estudiante, ambos deben ser actores activos; por su parte, el estudiante debe realizar trabajo individual autónomo, ya sea en lecturas o en resolución de problemas, y el docente generando las secuencias de aprendizaje, utilizando plataformas de información, orientando y realizando el apoyo tutorial que requieran sus alumnos.

Para complementar, el Dr. Zabalza señala que la universidad y el docente no entregan al estudiante lo que el por sí mismo no pueda lograr o no posea, pues "la función del buen profesor o profesora no es otra que la de potenciar las capacidades de sus estudiantes, ayudarles a aprender, crear situaciones donde ellos y ellas, sus estudiantes, puedan construir sus saberes" (2013, p. I I).

\section{Procedimientos de evaluación}

Los procedimientos de evaluación constituyen un ámbito de aprendizaje y retroalimentación docente-estudiante, y a su vez obedece a procesos complejos, planificados y previamente acordados entre los estamentos formales e informados a los estudiantes: "la evaluación constituye una parte fundamental del hecho educativo y su uso se justifica en tanto en cuanto optimizamos su impacto sobre la calidad de los aprendizajes" (Mateo, 2006, p. I66).

La meta es que los estudiantes universitarios que se formen y egresen de esta universidad sean capaces de desempeñarse de buena manera en la profesión en la que se prepararon y que puedan responder a 
situaciones reales con implicancias disciplinares, valóricas y éticas. La formación debe centrarse en el saber, el saber hacer y el ser y convivir, lo que significa que nuestra preparación se puede visualizar en las formas de actuación de los estudiantes, eso le imprime un sello diferenciador a esta casa de estudio, o al menos es lo que se espera como equipo de carrera.

De acuerdo a las palabras del Dr. Zabalza, un profesor competente es aquel que cumple con los criterios básicos de las competencias: organización, presentación, relaciones interpersonales, orientación, evaluación. En otras palabras, esto quiere decir que:

- Es el que organiza, planifica, programa lo que va a realizar y lo presenta a los alumnos.

- Es el que se expresa y comunica de manera asertiva, clara y coherente.

- Es quien establece relaciones cordiales con sus estudiantes, genera un clima adecuado para el desarrollo de las clases.

- Es quien orienta, va más allá del aula, realiza tutorías y entrega tiempos a los alumnos como sujetos individuales.

- Es quien evalúa y genera instancias conocidas para ello.

Para profundizar y entender el proceso de evaluación desde la función de acompañamiento y guía, tomo lo dicho por Wood (I986) en relación a que el nuevo paradigma se podría definir por su acuerdo en los siguientes principios:

- Trata sobre el rendimiento del sujeto en relación consigo mismo, más que por referencia al rendimiento de otros.

- Busca comprobar la competencia más que la inteligencia.

- Tiene lugar bajo condiciones relativamente incontroladas y consecuentemente no produce datos conductualmente correctos.

- Busca la «mejor ejecución» más que la «típica ejecución».

- Permite una mirada más constructiva respecto de la evaluación, donde el objetivo es ayudar al sujeto más que sentenciarlo (Wood, I986, citado en Mateo, 2006, pp. I7I-I72)

Como síntesis temática, la docencia universitaria es un acto complejo y de alta responsabilidad, en el que la mayor importancia radica en 
planificar y organizar lo que se espera que el estudiante aprenda, utilizando estrategias innovadoras que sean significativas a la hora de finalizar los procesos de manera exitosa y efectiva, planteándose siempre desde el acompañamiento docente y focalizado en el aprendizaje del estudiante.

\section{Metodología}

Esta investigación se enmarca en un paradigma cualitativo, con la intención de comprender lo que está sucediendo en una realidad única e irrepetible, pues se busca indagar y describir las opiniones que tienen las estudiantes de un nivel y carrera en específico, en relación a una profesora en particular: "Se opta por una metodología cualitativa basada en la rigurosa descripción contextual de un hecho o una situación que garantice la máxima intersubjetividad en la captación de una realidad compleja mediante la recogida sistemática de datos (...) que haga posible un análisis interpretativo" (Pérez Serrano, 200I, p. 26).

Debido a los instrumentos de recogida de información y el tipo de análisis a realizar, tiene un carácter mixto, ya que los datos que permitirán responder a las preguntas y metas del estudio, serán extraídos de un cuestionario organizado en respuestas cerradas e índices numéricos con significado criterial, tipo Escala Lickert, para luego ser analizados cualitativamente; por tanto, se cumple con dos fases de análisis; cuantitativa y cualitativa, porque según lo que plantean Cook y Reichardt (I986), existen ventajas en la utilización conjunta de ambos métodos, ya que se produce "la vigorización mutua de los tipos de métodos. En un sentido fundamental, los métodos cualitativos pueden ser definidos como técnicas de comprensión personal, de sentido común y de introspección, mientras los métodos cuantitativos podrían ser definidos como técnicas de contar, de medir y de razonamiento abstracto" (Pérez Serrano, 200I, p. 62)

El diseño de investigación utilizado es el estudio de caso, ya que este tiene por objetivo comprender el significado de una experiencia, es decir, comprender cómo funcionan todas las partes juntas para formar 
un todo, cuyo escenario es la carrera de Pedagogía en Educación Diferencial, Mención Discapacidad Cognitiva y Alteraciones Severas del Desarrollo de la UCSH, específicamente el nivel 700, equivalente al inicio de $4^{\circ}$ año de formación. Según Hernández et al. (2003), indica que los actores representan a un subgrupo de la población de interés sobre la cual se recolectan datos, y que tiene que definirse de antemano con precisión; por tanto, los actores principales involucrados en la investigación son I5 estudiantes, quienes componen la totalidad del grupo, equivalente al 100\%, cuyas edades varían entre los 20 y los 26 años y que están cursando por primera vez la asignatura, pero además previamente han cursado otras 2 o 3 actividades nucleares con la investigadora.

\section{Recolección de datos}

Los mecanismos para recabar los datos corresponden a:

- Cuestionario: que permita conocer las opiniones de las estudiantes en torno a 5 aspectos centrales: organización curricular, dominio conceptual y teórico, estrategias utilizadas, procedimientos de evaluación, además de la interacción docente-alumno. El cuestionario a aplicar fue publicado por la Universidad Arturo Prat de Chile ("Valoración del desempeño docente de cátedra”), y de acuerdo con la distribución de temas se ajusta a los requerimientos de esta investigación

- Revisión bibliográfica exhaustiva, para obtener los elementos teóricos que sustentan el estudio a partir de documentación trabajada en el Máster, pertinente a la temática abordada en la investigación: "la recogida de datos mediante el estudio de documentos sigue el mismo esquema de razonamiento que la observación o la entrevista. Hay que tener la mente organizada” (Stake, 1999, p. 66)

Como medios de registro, que entregan orden, sistematicidad y rigurosidad científica a los datos recogidos, la opción de organización de resultados es la siguiente: 
- Tabulación de respuestas gráficos: todas las respuestas dadas en el cuestionario serán traspasadas a cantidades para luego ser graficadas.

- Reducción de respuestas escritas: todas las respuestas escritas serán recogidas en un mismo registro, para luego darles los énfasis según las dimensiones de análisis.

- Matriz de constructo: con esta matriz se espera buscar la coherencia entre las respuestas de las estudiantes con las dimensiones de análisis.

- Matriz de triangulación teórica: permitirá vaciar los datos recogidos, en relación a las dimensiones, para cruzarlos con la teoría previamente seleccionada y con ello obtener conclusiones preliminares.

Según lo planteado por Gloria Pérez Serrano, la triangulación teórica "se basa en la preferencia de teorías alternativas o competitivas sobre la utilización de un solo punto de vista. Se emplean diferentes perspectivas coincidentes en un mismo objetivo" (2007, p. 83). Es por ello que en la presente investigación se realiza una matriz que incluye referentes teóricos, datos recogidos y la interpretación crítica de ellos por parte de la investigadora.

\section{Conclusiones}

Se logra culminar el estudio teniendo claro que es una investigación muy focalizada, debido a que el número de participantes es muy reducido; por ello se reafirma que este estudio de caso no pretende generalizar nada, solo reconocer la situación que se vive tal y cual en un momento determinado y con un grupo intencionado de actores que conforma uncurso completo de I5 estudiantes; por tanto, se cuenta con el I00\% de las respuestas. Para mayor detalle, se concluye a partir de los objetivos planteados en el inicio del estudio:

Objetivo específico I: Identificar los elementos organizativos de la estructura curricular que perciben las estudiantes de la asignatura.

Este objetivo logró ser cumplido, ya que de acuerdo a las respuestas de las alumnas y a los análisis realizados, la profesora en cuestión, 
da cumplimiento a la entrega de un programa con los contenidos de la asignatura, señala los tipos de procedimientos evaluativos y sus ponderaciones. Evidencia claramente la planificación de las clases y actividades, con una organización y preparación acorde a los contenidos y requerimientos de las estudiantes. Además de la entrega de los materiales pertinentes a la asignatura, recomienda alternativas de apoyo para favorecer el aprendizaje; finalmente hay consenso en que la profesora da cumplimiento a lo programado en la asignatura relevando que las responsabilidades de la formación corresponden a "un conjunto de acciones planificadas sistemáticamente en el tiempo que se llevan a cabo para lograr un perfeccionamiento en cualquiera de las funciones profesionales" (Mayor, 20I0, p. I)

Objetivo específico 2: Determinar cuál es el nivel de dominio teórico que identifican las estudiantes en la docente que desarrolla la asignatura.

Este objetivo también fue cumplido en su totalidad, pues según opinión de las estudiantes la docente tiene un muy buen nivel de dominio conceptual, disciplinar, técnico, actualizado y pertinente a la asignatura y la carrera en general, además les entrega insumos fundamentales para el desarrollo de su posterior práctica profesional, ya que muchos aspectos teóricos se desarrollan en terreno bajo la orientación de la profesora; por tanto, es posible señalar que "la universidad no es ya la única depositaria de la información, ni es tampoco la única capaz de difundirla, ni siquiera la que posee mayores capacidades expresivas y técnicas para hacerlo” (Sola, 2004, p. 94), pues $\sin$ los aprendizajes no se experimentan desde la realidad, se pueden olvidar y dejar vacíos conceptuales importantes para el futuro desempeño laboral.

Objetivo específico 3: Describir las estrategias de enseñanza que identifican las estudiantes en la práctica pedagógica de la docente de la asignatura.

El tercer objetivo también está cumplido en su casi totalidad, pues se han podido determinar algunas estrategias utilizadas por la docente, como son la participación activa, los trabajos colaborativos o en grupo, la discusión y reflexión permanente, la exploración de conocimientos, 
la creación de un clima propicio para aclarar dudas, basado en la confianza, empatía y ponderación de todos los estudiantes, sin dejar de lado las clases expositivas de contenido. A pesar de no ser una estrategia, las estudiantes valoran la disposición de la docente para aclarar dudas o apoyar en los aprendizajes, tanto dentro como fuera de la sala de clases: "la implantación de un sistema de tutorías en el marco de la enseñanza universitaria ha de constituir un factor clave que ayude a dinamizar este nuevo modelo formativo, cuyo eje central lo constituye el proceso de aprendizaje del alumnado y la ayuda metodológica que recibe del profesorado" (Álvarez \& González, 2005, p. 2).

Aquellos aspectos que deben ser mejorados se relacionan con la búsqueda de información autónoma, fomentar el autoaprendizaje y aumentar el uso de recursos adicionales a los habituales usados en clases. Según Álvarez y González, "el profesorado ha de asumir este nuevo reto, renunciando al papel protagonista que ha tenido en la formación universitaria como experto en contenidos, para centrar sus esfuerzos en facilitar el logro de las competencias y estimular el aprendizaje autónomo del alumnado".

Objetivo específico 4: Caracterizar los diferentes elementos evaluativos que identifican las estudiantes, como utilizados por la docente de la asignatura.

Este objetivo fue plenamente cumplido desde las respuestas obtenidas en el cuestionario, ya que la totalidad de las estudiantes consideran que las evaluaciones que realiza la docente son coherentes con los contenidos de la asignatura, además de permitir realizar un proceso de evaluación reflexivo y crítico, con un alto grado de responsabilidad personal, pues ellas también tienen participación en el tipo de evaluación a utilizar. Se destaca además que existe un proceso abierto de revisión de las evaluaciones, lo que permite la retroalimentación de las mismas, generando una conversación activa, crítica y facilitadora de nuevos aprendizajes. Según lo que plantean García \& Troyano, la evaluación puede responder a las necesidades emergentes de los estudiantes a lo largo de su formación académica, por ello puede permitir "dotar al alumnado de estímulos para el desarrollo de la reflexión, el diálogo, la autonomía y la crítica en el ámbito académico" (2009, p. I). 
Objetivo específico 5: Identificar los tipos de interacción que se establecen entre docente y alumnos más allá de la docencia de aula.

Este objetivo fue logrado en su totalidad y es uno de los que más llamaron mi atención por la cantidad de respuestas positivas y valoración a la docencia que realizo. De acuerdo entonces a las respuestas de las estudiantes, la interacción que se establece es a través de la comunicación directa, fluida y con alto grado de confianza, tanto dentro de la sala como fuera de ella. Se destaca la importancia que las estudiantes otorgan a la flexibilidad de la docente, la empatía, comprensión y acompañamiento, lo que genera vínculos cercanos en comparación con el resto de los docentes.

Por otra parte, se señala que la profesora logra crear una atmósfera de tolerancia y participación en clases, generando buenas relaciones entre el grupo curso en su totalidad; esto favorece el desempeño y aprendizaje de todas las alumnas, pues actúan como un gran equipo.

Finalmente, las estudiantes señalan que existe imparcialidad en el trato de la docente hacia las alumnas, lo que por supuesto evita problemas de roce dentro del grupo curso y les entrega oportunidades igualitarias de acceso y participación. Ello ya depende de cada una de ellas, pues, el profesor presta "una función básica de apoyo en los procesos de formación: prestar guía y apoyo a los estudiantes y ayudarles a resolver los problemas que se les vayan presentando tanto en relación a nuestra disciplina como en relación a la carrera en su conjunto" (García \&Troyano, 2009, p. 4).

Objetivo específico 6: Determinar aquellos aspectos susceptibles de mejorar a partir de la relación con la función tutorial del profesor en la universidad.

Luego de analizados todos los objetivos y matrices previas, puedo señalar que como docente debo poner mayor atención a la planificación: "Toda planificación debe partir del análisis de necesidades, concretar un plan de actuación (que además de explicitar objetivos, recursos, delimita el modelo tutorial y la distribución/coordinación de funciones) y establecer mecanismos de seguimiento y mejora” (Gairín et al., 2004, p. 2I) Esta tarea la 
realizo cotidianamente, interiorizándome primero del grupo curso asignado y de las asignaturas que ya han cursado como diagnóstico previo, pero no realizo un seguimiento sistemático, lo que sería un gran aporte a reconocer la trayectoria formativa de los estudiantes, detectando las mayores dificultades a las que se enfrentan.

Otro aspecto que debe claramente mejorar es la declaración abierta de la acción tutorial a realizar, considerándola como una innovación en mi docencia, lo que favorecerá a los estudiantes por las características de aprendizaje y desafíos que esta genera. "La tutoría, entendida como conjunto de actuaciones dirigidas a apoyar el pleno desarrollo personal y académico del estudiante universitario, se considera, cada vez más, como un indicador de calidad. Bien organizada, contribuye a mejorar la adaptación y rendimiento de los estudiantes a la vez que mejora la imagen pública de la universidad” (Gairín, Feixas, Franch, Guillamón \& Quinquer, 2004, p. 2I).

Finalizado el análisis de los objetivos específicos, es posible llegar a ver si se cumplió con:

Objetivo general: Analizar las opiniones vertidas por estudiantes que cursan $4^{\circ}$ año en Educación Diferencial de la UCSH, acerca de la docencia universitaria de la asignatura Propuesta de Intervención en la DI y su relación con la función tutorial del profesor en la universidad.

Desde mi opinión y por los resultados obtenidos y analizados, este estudio logró su objetivo, ya que a través del cuestionario se pudo recoger la opinión de las estudiantes en relación al problema planteado y, a su vez, reconocer la relación del desempeño de una docente determinada con la función tutorial del profesor en la universidad que se propone desde la teoría.

Claramente, con todo el desarrollo de la investigación y análisis previos realizados, puedo decir que las tutorías son una estrategia de innovación didáctica que puede generar grandes avances en la formación de nuestros estudiantes: "Mejorar la tutoría se sitúa en el marco de la mejora de la docencia universitaria y está en el contexto de las acciones que las universidades toman dirigidas a aumentar la calidad de los servicios que otorgan y su prestigio institucional" (Gairín et al., 2004, p. 22). 
Por otra parte, la acción tutorial permite que los estudiantes aprendan de manera autónoma, responsable, en donde el docente es solo quien guía u orienta su quehacer, pero que privilegia la reflexión y análisis crítico, trabajo en equipo y autoaprendizaje: "preparar al sujeto para el aprendizaje autónomo pero acompañado, dar más importancia al manejo de herramientas de aprendizaje que a la acumulación de conocimientos, equilibrio de la relación exigencias-apoyo para el aprendizaje” (García-Valcárcel, 2008, pp. 5-6).

Finalmente, dadas las características de los estudiantes que cumplen su proceso formativo en la UCSH y específicamente en la Carrera de Educación Diferencial, y de los beneficios que reporta la función tutorial, su puesta en marcha sería muy importante, pues podría "constituir un factor clave que ayude a dinamizar este nuevo modelo formativo, cuyo eje central lo constituye el proceso de aprendizaje del alumnado y la ayuda metodológica que recibe del profesorado. Ello va a requerir un cambio de mentalidad en buena parte del profesorado universitario que ha de redefinir su labor, haciendo que el péndulo oscile desde una práctica docente basada en la enseñanza a otra basada en el aprendizaje autónomo del alumnado" (Álvarez \& González, 2005, p. 2).

\section{Referencias bibliográficas}

Álvarez, P. R. y González, M. C. (2008). Análisis y valoración conceptual sobre las modalidades de tutoría universitaria en el espacio europeo de educación superior. Revista Interuniversitaria de Formación del Profesorado, 6I, 49-70.

Asensio, A., Carballo, R., García, M., Guardia, S. y García, N. (2005). La tutoría universitaria ante el proceso de armonización europea. Revista de Educación, 37, 189-210.

Bisquerra, R. (2009). Metodología de la Investigación Educativa. Madrid: Editorial La Muralla.

CPEIP/Mineduc. (2008). Marco para la Buena Enseñanza. Santiago, Chile: Maval.

Cisterna, F. (2005). Categorización y triangulación como procesos de validación del conocimiento en investigación cualitativa. Revista Theoria, I4, 6I-7I.

Escudero, T. (s/f). Evaluación de los aprendizajes en la universidad. Documento policopiado 
Flecha, R., García, C. y Melgar, P. (2004). El proceso educativo de convergencia europea, una mirada crítica. Revista Interuniversitaria de Formación del Profesorado, I8(3), 8I-89.

García, N., Asensio, I., Carballo, R., García, M., Guardia, S. (2005). La Tutoría Universitaria ante el proceso de armonización europea. Revista de Educación, 37, 189-210.

García, N. (2008). La función tutorial de la Universidad en el actual contexto de la Educación Superior. Revista Interuniversitaria de Formación del Profesorado, 6I, 2I-48.

García, F. J. y Troyano, Y. (2009)._El Espacio Europeo de Educación Superior y la figura del profesor tutor en la universidad. Revista de Docencia Universitaria, 3, 22-39.

García, A. (2008). La tutoría en la enseñanza universitaria y la contribución de las TIC para su mejora. Revista Electrónica de Investigación y Evaluación Educativa, RELIEVE, I4(2), I-I4. Recuperado de http://www.uv.es/RELIEVE/ vI4n2/RELIEVEvI4n2_3.htm

Gairín, J., Franch, J., Feixas, M., Guillamón, C. y Quinquer, D. (2004). Elementos para la elaboración de planes de tutoría en la universidad. Contextos Educativos, 6, 2I-42.

Gibbs, G. y Simpson, C. (2009). Condiciones para una evaluación continuada favorecedora del aprendizaje. Barcelona: Octaedro editores.

Hernández, R., Fernández, C., Baptista, P. (2006). Metodología de la investigación. México: McGraw Hill

Lázaro, A. (1997). La acción tutorial de la función docente universitaria. Revista Complutense de Educación, 8(I), 22-29.

Mateo, J. (2006). Claves para el diseño de un nuevo marco conceptual para la medición y evaluación educativas. Revista de Investigación Educativa, 24 (I), I65-I86.

Marcelo, C., Yot, C. y Mayor, C. (20II). Repositorio de diseños de aprendizaje para la enseñanza universitaria. Revista Científica de Edocomunicación, 19, 37-44.

Martínez, M. (2009). La orientación y la tutoría en la Universidad en el marco del Espacio Europeo de Educación Superior (EEES). Revista Fuentes, 9, 97-I07.

Mayor, C. (2010). Estrategias formativas/docentes. Sevilla: Editorial Universidad de Sevilla.

Meza, L. G. (2002). Metodología de la Investigación Educativa: Posibilidades de Integración. Comunicación. Revista Instituto Tecnológico de Costa Rica, I2(I), I-I3. 
Perea, M.V. y Cubo, S. (2010). Plan docente y tutorial en contextos virtuales en la universidad de Extremadura. Revista Española de orientación y Psicopedagogía, 2I(I), I3-24.

Rojas A. (2009). La investigación acción en el aula. Reflexiones de Profesionales de la Educación. Conferencia Iberoamericana de Ministros de Educación (pág. s/i). Junin, Perú: Organización de Estados Americanos.

Saravia, M. (20I I). Calidad del profesorado: un modelo de competencias académicas. Barcelona: Editorial Octaedro.

Sola, T. (2004). La formación del profesorado en el contexto del Espacio Europeo de la Educación Superior. Revista Interuniversitaria de Formación del Profesorado, I8(3), 9I-I06.

Pérez, G. (200I). Investigación Cualitativa. Retos e Interrogantes. Madrid: Editorial La Muralla, S.A.

Pérez, G. (2007). Investigación cualitativa, Retos e interrogantes. II Técnicas y análisis de datos. (4 $\left.4^{\mathrm{a}} \mathrm{E}\right)$. Madrid: Editorial La Muralla, S.A.

Stake, R. (1999). Investigación con estudio de casos. Madrid: Morata.

Stake, R. (2007). Investigación con estudio de caso. Madrid: Morata.

Zabalza, M. (2006). Competencias docentes del profesorado universitario: calidad y desarrollo profesional, Madrid: Narcea.

Zabalza, M. (2009). Diseño y Desarrollo Curricular. Madrid: Narcea.

Zabalza, M. (2009). Ser Profesor universitario hoy. Revista La Cuestión Universitaria, $5,69-81$.

Zabalza, M. (2013). Ser docente más que enseñarte. Revista de Docencia Universitaria, II(2), 3I-45.

\section{Documentos}

Carrera de Pedagogía en Educación Diferencial (2014). Informe Proceso de Autoevaluación CNA. Santiago: UCSH.

Ministerio de Educación de Chile. Documentos Prueba Inicia. Recuperado de: http://www.mineduc.cl/index2.php?id_seccion=4245\&id_ portal=798id_contenido $=20559$

Programa de la Actividad Curricular (2009). Formulario N B 4. Didoc Santiago: UCSH.

Secretaría de Marina, Armada de México. (2010). Manual para elaborar y evaluar trabajos de investigación. México. 